\title{
Postpartum Depressive Symptoms: Risks for Czech and Thai Mothers
}

\author{
Silva Hassert, $\mathrm{PhD}$ \\ Sharon Robinson Kurpius, PhD \\ Anusorn Payakkakom, PhD \\ Eliška Kodyšová, PhD
}

\begin{abstract}
Multiple psychosocial risk factors are linked to women's experience of postpartum depressive symptoms worldwide. This study focused on mothers in the Czech Republic $(n=126)$ and Thailand $(n=161)$, two countries where little research on mothers' experiences of postpartum depressive symptoms (PPDS) has been conducted. Measures included the Postpartum Social Support Questionnaire, Parenting Sense of Competence Scale, Quality of Relationships Inventory, Dysfunctional Attitude Scale-Form A-17, and Edinburgh Postnatal Depression Scale. Hierarchical linear regression analyses indicated that for Czech mothers, financial concerns, partner conflict, perfectionism, maternal efficacy, and parental support were significantly related to PPDS. For Thai mothers, only partner conflict and perfectionism were significantly related to PPDS. Cultural implications for childbirth educators are discussed in the context of these risk factors.
\end{abstract}

The Journal of Perinatal Education, 27(1), http://dx.doi.org/10.1891/1058-1243.27.1.38

Keywords: women, postpartum period, depressive symptoms, family life cycles

For childbearing women, postpartum depressive symptoms (PPDS) have become an international health concern. Women can experience PPDS beyond the first year after birth, which can be quite severe and debilitating (Vliegen, Casalin, \& Luyten, 2013). For mothers, not only can PPDS reduce the ability to carry out parenting tasks, they have also been linked with poorer developmental outcomes for children (Milgrom, Ericksen, Sved-Williams, \& Sutter-Dallay, 2016). Despite these negative consequences, many mothers do not report seeking any formal treatment (Callister, Beckstrand, \& Corbett, 2010; Marcus, 2009). International studies on women's experience of PPDS have been conducted; however, there are still countries and cultures where little research has examined specific risk factors that may play a role in the development of PPDS among women. We examined the risks for PPDS among mothers living in Thailand and the Czech Republic, two countries where there has 
been little research on this topic. Women who give birth in Thailand and the Czech Republic do so in different settings. Thus, the aim of this study was to draw attention to risk factors that may be universally present and relevant to mothers in both Eastern and Western cultures. It is believed that this study will be of interest to an international audience of childbirth educators as well as those working with mothers in the United States, where the population becomes more ethnically diverse each year.

The international prevalence of PPDS among mothers has been found to vary widely from $0 \%$ to $60 \%$ (Halbreich \& Karkun, 2006). While it was once questioned whether PPDS were solely experienced by mothers in westernized nations (Oates et al., 2004), it has since been welldocumented that mothers are affected by PPDS around the world (Halbreich \& Karkun, 2006). In their literature review of studies conducted in Asian countries between 1998 and 2008, Klainin and Arthur (2009) found that PPDS among mothers was as prevalent in Asian countries as in European countries. Reviewing 141 studies conducted in the United States, Australia, Canada, China, Finland, Iceland, Sweden, Turkey, and Malaysia, Beck (2008) identified lack of social support, low self-esteem, life stress, fatigue, prenatal depression and anxiety, depression history, difficult infant temperament, low socioeconomic status, single marital status, and unplanned/unwanted pregnancy as risk factors. Among a sample of Korean mothers at 24 weeks of pregnancy and at 1 week and 6 weeks of postpartum, Kim, Hur, Kim, Oh, and Shin (2008) found that only prenatal depression was a significant predictor of PPDS and that sociodemographic, obstetric, and psychological factors did not predict PPDS. In a study that included 237 Bahraini women at 8 weeks postpartum, Al Dallal and Grant (2012) did not find a relationship between women's PPDS and sociodemographic or obstetric risk factors, including maternal age, education, occupation, marital status, birth type, or breastfeeding status. Other studies conducted in the United States, Lebanon, and Japan, however, have found risk factors such as age, education, occupation, marital status, birth type, and breastfeeding status to be significantly related to PPDS among mothers (Chaaya et al., 2002; Nishioka et al., 2011; Segre, O'Hara, Arndt, \& Stuart, 2007).

We examined risk factors potentially playing a role in PPDS for Thai and Czech mothers.
International studies on women's experience of PPDS have been

conducted; however, there are still countries and cultures where

little research has examined specific risk factors that may play a

role in the development of PPDS among women.

In Thailand, traditional postpartum practices continue, despite significant social and economic transitions (Kaewsarn, Moyle, \& Creedy, 2003). Although it is understood that the birth of a child and the observance of such practices mark the transition to adulthood for women (Whittaker, 2002), little is known about the factors that place Thai mothers at risk for developing PPDS. Indeed, only two studies were found that examined PPDS among women in Thailand. For both studies, the prevalence of PPDS among Thai women at 6-8 weeks postpartum was found to be similar at 10 and $17 \%$, respectively (Liabsuetrakul, Vittayanont, \& Pitanupong, 2007; Limlomwongse \& Liabsuetrakul, 2006). Risk factors identified included anxiety, social support, religious beliefs, irritable mood related menstruation, pregnancy complications, and attitudes about pregnancy (Liabsuetrakul et al., 2007; Limlomwongse \& Liabsuetrakul, 2006).

Among the studies conducted within Europe, no study was found that specifically examined PPDS among Czech mothers. The Czech Republic has experienced significant economic and political transformation since the 1990s. It is particularly important to explore PPDS among Czech women given changes in family structure and health care (Bartonova, 2005). Understanding factors placing mothers at risk may lay the groundwork for earlier detection and interdisciplinary treatment for women. The current study was designed to address gaps in the literature by exploring whether general risk factors identified by previous researchers also are implicated in the development of PPDS among Thai and Czech mothers.

For mothers in many cultures, childbirth can create a stressful time in which psychological, cultural, and environmental risk factors leave some more susceptible to PPDS (Dudas et al., 2012). As such, we considered a variety of risk factors. Previous researchers have supported the role of the chosen risk factors for mothers internationally (e.g., Grote \& Bledsoe, 2007; Milgrom et al., 2008). In addition to demographic variables, clusters of intra- (personal characteristics) and interpersonal variables 
were examined. The selected intrapersonal risk factors included maternal efficacy, perfectionism, and dependency while the selected interpersonal risk factors included partner conflict and parental support. Based on previous research, we hypothesized that maternal efficacy, perfectionism, dependency, partner conflict, and parental support would predict PPDS among Thai and Czech mothers within 1 year of giving birth. Demographic variables including maternal age, infant age, parity status, breastfeeding status, income level/financial dissatisfaction, and education level were entered as control variables.

\section{METHOD}

\section{Participants and Procedure}

After Institutional Review Board approval was obtained from the institution with which the leading authors were affiliated, mothers living in the Czech Republic and Thailand were recruited to participate in this study. A power analysis, using the $G^{\star}$ Power computer program (Erdfelder, Faul, \& Buchner, 1996), indicated that a minimum of 123 participants was required per sample (effect size of .15 and power of .08). In the Czech Republic, mothers who had given birth within a 1-year period were recruited from a large maternity hospital outside of Prague. A recruitment script was provided to staff clinicians who notified women after giving birth about the study. Within 1 month of their delivery, mothers who expressed interest in participating were emailed a link to the survey questionnaire. The informed consent and survey were available to women online, and taking the survey was considered consent to participate. Although 146 mothers initially completed the study survey, 20 were omitted from the analyses due to significant missing data (one or more study measures left blank). For the remaining 126 study participants, age ranged from 22 to 39 years $(M=30.30, S D=3.99)$. The majority were married or in a committed relationship $(n=$ $123,98.4 \%)$, and approximately half ( $n=69 ; 54.8 \%)$ were multiparious. Thirteen $(10.3 \%)$ reported having a prior diagnosis of depression, and 18 (14.3\%) reported having sought professional treatment for depressive symptoms.

In Thailand, mothers were recruited from state hospitals and medical settings in metropolitan and rural areas. All participants had returned to the hospitals and medical settings to receive vaccinations for their infants. Following a verbal script, clinicians invited mothers to participate in the study. Those who chose to participate read the recruitment letter and signed the informed consent form. A paper version of the study survey was completed in the waiting rooms. Of the 225 Thai mothers who were administered the survey, 163 completed and returned the survey; however, 2 participants were deleted from the sample due to being under 18 years old. For the 161 participants, age ranged from 18 to 35 years $(M=$ $25.83, S D=3.46)$. The majority of participants were married or in a committed relationship ( $n=143$, $88.8 \%)$. Slightly less than half of the mothers ( $n=$ $70 ; 44.7 \%)$ were multiparious. Three (1.9\%) participants reported having a prior diagnosis of depression, and two (1.2\%) reported having previously sought professional treatment for depressive symptoms.

\section{MEASURES}

The measures were translated from English to Czech and Thai through a process of translation and backtranslation. In each country, bilingual speakers modified the questionnaire until a consensus was reached about equivalence with the English version to ensure accuracy of the wording and meaning of items. Information on the demographic form asked for maternal age, marital status, age(s) and number of children, employment status, financial concerns or income level, and education level. Mothers were asked whether they had been previously diagnosed with depression. If they responded "yes," they were asked to report whether they had sought professional help.

\section{Dependency and Perfectionism}

Dependency and perfectionism were measured using the Dysfunctional Attitude Scale-Form A-17 (DAS-A-17; de Graaf, Roelofs, \& Huibers, 2009). The DAS-A-17 was designed to assess the presence and intensity of negative thinking styles that have been related to depression, namely dependency and perfectionism (de Graaf et al., 2009). Women rated items on a scale of 1 (fully disagree) to 7 (fully agree). Dependency, defined as a need for approval from others, was measured by the 6-item Need for Approval subscale. A sample item from the dependency subs cale includes, "My value as a person depends greatly on what others think of me." Summing the responses to the six items yielded a score ranging from 6 to 42, with higher scores reflecting more dependency. Perfectionism, defined 
as "concern about being negatively evaluated by others on the basis of performance", was measured by the 11-item Perfectionism/Performance Evaluation subscale. A sample item from the perfectionism subscale includes, "If I fail partly, it is as bad as a complete failure." Summing the responses across the 11 items yielded a score ranging from 11 to 77, with higher scores reflecting more perfectionism. The DAS A-17 has established adequate reliability and validity. de Graaf et al. (2009) found a moderate correlation between the DAS-A-17 and the Diagnostic Inventory for Depression, a measure of depression severity. For de Graaf et al.'s (2009) sample of 8,960 participants, Cronbach's for dependency, perfectionism, and total score were reported to be $.81, .90$, and .91 , respectively. In the current study, Cronbach's alphas for the dependency subscale were .82 for Czech and .89 for Thai women. For the perfectionism subscale, Cronbach's alphas were .92 and .90 for Czech and Thai women, respectively.

\section{Parental Support}

Support received from a mother's own parents (maternal grandparents of the infant) was measured using the 11-item Parent Support subscale from the Postpartum Social Support Questionnaire (PSSQ; Hopkins \& Campbell, 2008). Each item was rated on a scale ranging from 1 (almost never) to 6 (very often). A sample item includes, "How often do your parents help with the baby?" Summing ratings across the 11 items created a total score ranging from 11 to 66 , with higher scores indicating more parental support. Hopkins and Campbell (2008) found that the PSSQ yielded strong internal validity and testretest reliability when administered to mothers at 2 , 4,6 , and 12 months postpartum. Cronbach's alphas were .90 and .94 for Czech and Thai participants, respectively.

\section{Maternal efficacy}

Maternal efficacy, defined as a mother's sense of personal competence, was measured using the 8-item Efficacy subscale of the Parenting Sense of Competence Scale (PSOC; Gibaud-Wallston \& Wandersman, 1978). Originally designed to assess parental feelings of competence with their infants, the PSOC has been used internationally with mothers in the postpartum period (e.g., Caramlau, Barlow, Sembi, McKenzie-McHarg, \& McCabe,
2011; Gao, Chan, \& Sun, 2012), and satisfactory reliabilities for the Efficacy subscale have been reported. Each item was rated on a scale ranging from 1 (strongly disagree) to 6 (strongly agree). A sample item includes, "Being a parent is manageable, and any problems are easily solved." Summed ratings ranged from 8 to 48 , with higher total scores indicating greater maternal efficacy. Cronbach's alphas were .83 and .78 for Czech and Thai mothers, respectively.

\section{Partner Conflict}

The 12-item Conflict subscale of the Quality of Relationships Inventory (QRI; Pierce, Sarason, \& Sarason, 1991) was used to measure partner conflict. Pierce, Sarason, and Sarason (1991) defined relationship conflict as the extent to which a close relationship is a source of ambivalence and conflict. The current study focused on mothers' relationships with their spouses/partners. Items such as "How often do you have to work hard to avoid conflict with this person?" were rated on a scale of 14 . Responses across the 12 items were summed, with higher scores indicating more partner conflict. Pierce et al. reported reliabilities for the Conflict subscale with Cronbach's alphas ranging from .88 to .91 . The QRI has been used in previous studies of women's PPDS (Dennis \& Ross, 2006; Hassert, Kurpius, \& Tracey, 2015). Cronbach's alphas were .90 for Czech and .88 for Thai mothers.

\section{Postpartum Depressive Symptoms}

PPDS were assessed using the 10-item Edinburgh Postnatal Depression Scale (EPDS; Cox, Holden, \& Sagovsky, 1987). A sample item includes, "I have looked forward with enjoyment to things." Each item was rated on a 4-point scale ranging from 0 to 3 based on how the mother had felt in the previous 7 days. Total scores range from 0 to 30, with higher scores reflecting the presence of more PPDS. One of the most commonly used measures of postpartum depression, the EPDS has been translated into many languages. It has been used in the majority of studies that have examined PPDS worldwide, including a few studiesstudies with women in Thailand and the Czech Republic (Pitanupong, Liabsuetrakul, \& Vittayanont, 2007Takács, Smolík, Mlíková Seidlerová, Čepický, \& Hoskovcová, 2016. Cronbach's alphas for the current study were .86 and .74 for Czech and Thai mothers, respectively. 


\section{DATA ANALYSIS}

Separate hierarchical linear regression analyses were conducted to evaluate the relationship among the risk factors and PPDS for each sample. For both samples, the outcome, PPDS, was entered as a continuous variable assessed by the total score on the EPDS. Since only a small number of Czech and Thai mothers reported either prenatal depressive symptoms or having prior depressive symptoms, neither of these variables were included into the analyses.

To control for demographic risk factors among the Czech participants, demographic variables were categorized and entered in step one of the regression analysis. The demographic variables categorized into two levels were maternal age (22-30 years and 31-39 years), infant age (less than 6 months or 6-12 months), parity status (primarious or multiparious), and current breastfeeding status (yes or no). Due to cultural consideration, income was not asked directly to Czech mothers; rather, they rated their level of dissatisfaction with their current financial situation from 1 to 4 , with 4 reflecting more financial dissatisfaction. Level of education included five levels (less than high school diploma, high school diploma, some college, college degree, or graduate degree). In the second step, the three intrapersonal risk factors (dependency, perfectionism, and maternal efficacy) were entered. The final step entered the interpersonal risk factors (partner conflict and parental support) into the regression analysis.

A parallel procedure was followed for Thai participants. Demographic variables were controlled for by entering them as step one of the analysis. These variables included maternal age (18-25 years and 26-35 years), infant age (less than 6 months or 6-12 months), parity status (primarious or multiparious), and breastfeeding (yes or no), each consisting of two levels. Income consisted of five levels and education consisted of four levels. In the second step, the intrapersonal risk factors (dependency, perfectionism, and maternal efficacy) were entered. The interpersonal risk factors (partner conflict and parental support) were entered in the final step.

\section{RESULTS}

Examination of the total EPDS scores indicated that 29 Czech mothers (23\%) had an EPDS score of $\geq 12$, which has been used as a cut-off score to identify the presence of major depression (Gaillard, Le Strat, Mandelbrot, Keïta, \& Dubertret, 2014). For the sample of Czech mothers, when demographics were entered in the first step of the hierarchical regression analysis, these variables accounted for only $12.8 \%$ $(F=2.72, d f=6,111, p=.017)$ of the variance in PPDS. The beta weights indicated that financial dissatisfaction was the only significant predictor $(\beta=$ $.331, t=3.60, p=.000,95 \% \mathrm{CI}=1.00,3.43)$ in the first step. In the second step, the intrapersonal factors enhanced the regression equation. The $\Delta R^{2}$ was .388 $(\Delta F=28.89, d f=3,108, p=.000)$. Of the intrapersonal variables, perfectionism $(\beta=.493, t=$ $5.85, p=.000,95 \% \mathrm{CI}=.131, .265)$ and maternal efficacy $(\beta=-.231, t=-3.12, p=.002,95 \% \mathrm{CI}=$ $-.307,-.068)$ significantly predicted PPDS. Finally, when the last two interpersonal variables were added in the third step, these variables also enhanced the accounted for variance $\left(\Delta R^{2}\right.$ was $.028, \Delta F=3.32$, $d f$ $=2,106, p=.04)$. In this third step, the beta weight for partner conflict was .192 $(t=2.54, p=.013,95 \%$ $\mathrm{CI}=.035, .286)$ and for parental support the beta weight was $-.215(t=-2.95, p=.004,95 \% \mathrm{CI}=$ $-.292,-.057)$. The final model accounted for $49.7 \%$ of the total variance in PPDS among the sample of Czech mothers. PPDS were related to more financial dissatisfaction, perfectionism, and partner conflict, and less maternal efficacy and parental support. The results of the regression analysis for Czech participants are presented in Table 1.

Among the Thai mothers, 58 (36\%) had a total EPDS score of $\geq 12$, the established cut-off score (Gaillard, Le Strat, Mandelbrot, Keïta, \& Dubertret, 2014). The first step of the hierarchical regression analysis included demographic variables that accounted for only $3.0 \%$ of the variance in PPDS $(F=.81, d f=6,154, p=.56)$. In the second step, the intrapersonal factors were added to the regression equation. The $\Delta R^{2}$ was $.169(\Delta F=10.62, d f$ $=3,151, p=.000)$. Of the intrapersonal variables, only perfectionism significantly predicted PPDS $(\beta=.369, t=3.11, p=.002,95 \% \mathrm{CI}=.054, .240)$. Finally, when the two interpersonal variables were added, they enhanced the accounted for variance $\left(\Delta R^{2}\right.$ was $\left..087, \Delta F=9.06, d f=2,149, p=.000\right)$. The beta weight for perfectionism was $.354(t=$ $3.12, p=.002,95 \% \mathrm{CI}=.051, .230)$ and for partner conflict was .302 $(t=4.23, p=.000,95 \% \mathrm{CI}=$ $.130, .357)$. The final model accounted for $23.3 \%$ of the variance in PPDS among the sample of Thai mothers. PPDS were significantly related to more perfectionism and partner conflict. Table 2 presents these regression results. 
TABLE 1

Regression Analysis for Variables Predicting Postpartum Depression Among Czech Mothers

\begin{tabular}{|c|c|c|c|c|c|c|}
\hline Step and Variable & $\beta$ & $R^{2}$ & Adj. $R^{2}$ & $\Delta R^{2}$ & $\Delta F$ & $p$ \\
\hline Step 1 & & & .081 & .128 & 2.717 & .017 \\
\hline \multicolumn{7}{|l|}{ Demographic variables } \\
\hline 1. Maternal age & .080 & & & & & .405 \\
\hline 2. Breastfeeding status & .043 & & & & & .640 \\
\hline 3. Financial dissatisfaction & $.331^{* *}$ & & & & & .000 \\
\hline 4. Infant age & -.056 & & & & & .534 \\
\hline 5. Parity status & .072 & & & & & .457 \\
\hline 6. Education & .016 & & & & & .870 \\
\hline Step 2 & & & .476 & .388 & 28.889 & .000 \\
\hline \multicolumn{7}{|l|}{ Demographic variables } \\
\hline 1. Maternal age & .077 & & & & & .293 \\
\hline 2. Breastfeeding status & .133 & & & & & .062 \\
\hline 3. Financial dissatisfaction & $.261^{* *}$ & & & & & .000 \\
\hline 4. Infant age & -.042 & & & & & .544 \\
\hline 5. Parity status & .070 & & & & & .341 \\
\hline 6. Education level & .001 & & & & & .990 \\
\hline \multicolumn{7}{|l|}{ Intrapersonal variables } \\
\hline 7. Perfectionism & $.493^{* *}$ & & & & & .000 \\
\hline 8. Maternal efficacy & $-.231^{* *}$ & & & & & .002 \\
\hline 9. Dependency & .051 & & & & & .544 \\
\hline Step 3 & & & .497 & .028 & 3.315 & .040 \\
\hline \multicolumn{7}{|l|}{ Demographic variables } \\
\hline 1. Maternal age & .062 & & & & & .390 \\
\hline 2. Breastfeeding status & .128 & & & & & .070 \\
\hline 3. Financial dissatisfaction & $.217^{* *}$ & & & & & .005 \\
\hline 4. Infant age & -.059 & & & & & .381 \\
\hline 5. Parity status & .072 & & & & & .328 \\
\hline 6. Education level & .003 & & & & & .969 \\
\hline \multicolumn{7}{|l|}{ Intrapersonal variables } \\
\hline 7. Perfectionism & $.467^{* *}$ & & & & & .000 \\
\hline 8. Maternal efficacy & $-.215^{* *}$ & & & & & .004 \\
\hline 9. Dependency & .032 & & & & & .697 \\
\hline \multicolumn{7}{|l|}{ Interpersonal variables } \\
\hline 10. Parental support & .081 & & & & & .266 \\
\hline 11. Partner conflict & $.192^{*}$ & & & & & .013 \\
\hline
\end{tabular}

${ }^{*} p \leq .05 .{ }^{* *} p \leq .01$.

\section{DISCUSSION}

An EPDS score of 12 or greater, indicating PPDS, was reported in $23 \%$ of Czech and $36 \%$ of Thai mothers. These percentages are higher than the 10\%-15\% of mothers in the United States who experience PPDS (Lanes, Kuk, \& Tamim, 2011). They are consistent with the variability that has been found for rates of PPDS worldwide (Halbreich \& Karkun, 2006). In the current study, nearly twice as many Thai mothers reported PPDS than was previously reported by Limlomwongse and Liabsuetrakul (2006) in their study that included Thai mothers at 6-8 weeks postpartum. For the sample of Czech women, no prior study was found that allowed for a comparison in the rate of PPDS. Past researchers have found that cultural differences, size of sample, time since birth, and EPDS cut-off scores have contributed to the differences in reported PPDS rates (Dindar \& Erdogan, 2007). The higher rates for the women in the current study could perhaps be related to the demographic characteristics of the sample which included mothers up to 12 months after giving birth. Another factor to consider is that most women had not received prior treatment for depressive symptoms (only 1\% of Thai and 14\% of Czech mothers reported having sought formal help). Given the number of mothers in this study reporting EPDS

Twenty three percent of Czech and $36 \%$ of Thai mothers reported an EPDS score of 12 or greater, indicating PPDS. These percentages are higher than the 10\%-15\% of mothers in the United States who experience PPDS. 
TABLE 2

Regression Analysis for Variables Predicting Postpartum Depression Among Thai Mothers

\begin{tabular}{|c|c|c|c|c|c|c|}
\hline Step and Variable & $\beta$ & $R^{2}$ & Adj. $R^{2}$ & $\Delta R^{2}$ & $\Delta F$ & $p$ \\
\hline Step 1 & & .031 & -.007 & .031 & .817 & .558 \\
\hline \multicolumn{7}{|l|}{ Demographic variables } \\
\hline 1. Maternal age & -.126 & & & & & .149 \\
\hline 2. Breastfeeding status & -.049 & & & & & .662 \\
\hline 3. Income & .125 & & & & & .131 \\
\hline 4. Infant age & .099 & & & & & .385 \\
\hline 5. Parity status & .030 & & & & & .731 \\
\hline 6. Education & -.074 & & & & & .379 \\
\hline Step 2 & & .200 & .152 & .169 & 10.623 & .000 \\
\hline \multicolumn{7}{|l|}{ Demographic variables } \\
\hline 1. Maternal age & -.114 & & & & & .157 \\
\hline 2. Breastfeeding status & -.052 & & & & & .617 \\
\hline 3. Income & .116 & & & & & .129 \\
\hline 4. Infant age & .080 & & & & & .444 \\
\hline 5. Parity status & -.020 & & & & & .808 \\
\hline 6. Education level & .010 & & & & & .903 \\
\hline \multicolumn{7}{|l|}{ Intrapersonal variables } \\
\hline 7. Perfectionism & $.369^{* *}$ & & & & & .002 \\
\hline 8. Maternal efficacy & -.127 & & & & & .091 \\
\hline 9. Dependency & .043 & & & & & .718 \\
\hline Step 3 & & .287 & .234 & .087 & 9.077 & .000 \\
\hline \multicolumn{7}{|l|}{ Demographic variables } \\
\hline 1. Maternal age & -.076 & & & & & .325 \\
\hline 2. Breastfeeding status & -.036 & & & & & .716 \\
\hline 3. Income & .104 & & & & & .152 \\
\hline 4. Infant age & .096 & & & & & .337 \\
\hline 5. Parity status & -.071 & & & & & .366 \\
\hline 6. Education level & .026 & & & & & .731 \\
\hline \multicolumn{7}{|l|}{ Intrapersonal variables } \\
\hline 7. Perfectionism & $.354^{* *}$ & & & & & .002 \\
\hline 8. Maternal efficacy & -.083 & & & & & .262 \\
\hline 9. Dependency & .070 & & & & & .539 \\
\hline \multicolumn{7}{|l|}{ Interpersonal variables } \\
\hline 10. Parental support & -.003 & & & & & .969 \\
\hline 11. Partner conflict & $.302^{* *}$ & & & & & .000 \\
\hline
\end{tabular}

${ }^{*} p \leq .05 .{ }^{* *} p \leq .01$.

scores of $\geq 12$, it is important to understand the risk factors playing a role in the emotional well-being of mothers in both countries. Czech women who reported more financial dissatisfaction, perfectionism, and partner conflict and less maternal efficacy and parental support were at greatest risk for PPDS. Among Thai women, only perfectionism and partner conflict were significant risk factors.

For mothers in both countries, perfectionism played a role in the development of PPDS. Women

\section{For mothers in both countries, perfectionism played a role in the} development of PPDS. Women who reported more perfectionism, or fear of being negatively evaluated by others, were found to be at greatest risk. who reported more perfectionism, or fear of being negatively evaluated by others, were found to be at greatest risk. Across cultures, the postpartum period has been perceived as a time when women rely more on others, commonly their spouse and family, for support and assistance caring for their infant and other daily tasks (Kaewsarn et al., 2003). For mothers who struggle with perfectionism, perhaps just being a "good" mother is not enough. Concerns about making mistakes or fear of judgment may cause them to feel more overwhelmed by maternal responsibilities. Such worries about needing to be perfect may be reflected in more PPDS. Given the nature of the current study, it is difficult to know whether mothers who have concerns about perfectionism are more prone to depressive symptoms or whether mothers who 
are already feeling depressed are more sensitive to judgments from others. The finding that perfectionism was a risk factor among both samples of women is important in that future research can incorporate other intrapersonal traits to study PPDS risks more comprehensively. In light of evidence that mothers who report higher levels of perfectionism after giving birth are at greater risk for developing depression years later (Vliegen et al., 2010), the role of perfectionism should continue to be explored with postpartum women in other countries.

In addition to perfectionism, partner conflict was found to be a significant risk factor for both Czech and Thai mothers. Mothers who reported more partner conflict also reported greater PPDS. This finding aligns with previous research (e.g., Milgrom et al., 2008) and highlights the role that significant others, particularly a partner or spouse, can have in women's emotional and physical wellbeing. Whereas several researchers have focused on receiving social support (Xie, He, Koszycki, Walker, \& Wen, 2009), few have examined the specific role of partner conflict (Dennis \& Ross, 2006; Hassert \& Robinson Kurpius, 2011). Conflict is likely to produce greater emotional distance between women and their partners. Women who experience more conflict with their partners may be at a disadvantage as they are likely to also experience less partner support. This study extends past research by suggesting that partner conflict, in addition to support, has implications for understanding women's PPDS.

For Czech mothers, financial dissatisfaction, maternal efficacy, and parental support were also significant. Some research suggests that it is common for women to experience depression that occurs because of interpersonally related life events, and that depression may contribute to the perpetuation of perceived stress (Hammen, 2003). Financial problems are one such source of life stress. For many families, the birth of a child poses an added financial challenge related to costs of caring for an infant, raising a child, and concerns about changes in work schedule to meet childcare responsibilities. Hraba, Lorenz, and Pechačová (2000) found that the economic pressure placed on Czech families following government reform led to more irritability, tension, and depression among couples. Economic troubles have continued to persist for some families (Hraba, Lorenz, \& Pechačová, 2000) and may have affected some of the women participating in the current study. As the perception of one's financial situation predicts emotional distress (Grote \& Bledsoe, 2007), future research should examine how factors such as how parental support may buffer the link between financial dissatisfaction and PPDS. Interestingly, no relationship between income level and PPDS was found for Thai mothers. It is possible that the perception of being financially burdened, rather than women's actual reported income level, is a more important factor and should be further teased apart.

This study also found a relationship between maternal efficacy and PPDS for Czech mothers. Numerous biological, emotional, and social changes simultaneously occur for women in the perinatal period. It seems intuitive that women who feel less efficacious about mothering may also feel they are ill-prepared to meet the parenting demands placed upon them, while those who feel more confident may feel better equipped to meet maternal demands (Haslam, Pakenham, \& Smith, 2006). In the current study, maternal efficacy and perfectionism were negatively correlated $(r=$ $.320, p=.000)$ for Czech mothers, suggesting that women who felt more efficacious may also have been less perfectionistic. The same relationship was not found for Thai mothers. For these mothers, there was also no significant relationship between maternal efficacy and perfectionism, and maternal efficacy was not related to PPDS. This suggests that maternal efficacy may be a more important predictor for women in some cultures, but not in others. Choi, Kim, Ryu, Chang, and Park (2012) found a significant negative correlation between PPDS and parental self-efficacy for native Korean mothers but no relationship for immigrant Vietnamese mothers living in South Korea. Traditional cultural beliefs and birthing practices provide an important context for women (Callister, Beckstrand, \& Corbett, 2010; Gulamani, Premji, Kanji, \& Azam, 2013). For Thai mothers, perhaps other more relevant factors posed a greater risk for PPDS. It is also possible that cultural rituals and the cultural norm of receiving help from extended family in Thailand-a factor that was not examined in the current study-played a protective role in buffering against low maternal efficacy. As the relationships among maternal efficacy, perfectionism, and PPDS are preliminary, future research should address these relationships with larger samples. 
TABLE 3

- Nearly $23 \%$ of Czech and 36\% of Thai mothers experienced PPDS within 12 months after giving birth. Many reported they had not received professional help for their mental health (1\% of Thai mothers and $14 \%$ of Czech mothers). Childbirth educators caring for mothers in these countries should be aware that women who experience PPDS may not seek help formally and should discuss symptoms while screening for potential risk factors.

- Perfectionism and partner conflict were significant risk factors in the development of PPDS for Czech and Thai women. Less financial satisfaction, maternal efficacy, and parental support were also significant for Czech women. Regardless of the country in which a new mother lives, childbirth educators play an important role in helping women become aware of PPDS risk factors that may assist in earlier detection and treatment of PPDS. Understanding the overlap in risk factors is crucial for women worldwide.

- Prevention programs implemented in one country may be helpful to childbirth educators helping mothers living elsewhere in the world. Understanding cultural context and developing culturally targeted interventions is the key to bolstering maternal well-being while reducing international health-care costs.

In this study, we found less parental support to be a risk factor for Czech, but not for Thai, women. Sixty-one percent of the Czech women reported that their mother or mother-in-law provided the most help in caring for their infant, compared with 79\% of the Thai women. While almost four of five Thai mothers reported having support from their own parents, there was more variation in the individuals who were providing the most support for Czech mothers. Few women in either sample reported that either their fathers or father-in-laws helped with infant care (only $6 \%$ for Czech and $4 \%$ for Thai). Given the link between social support and PPDS (Dennis \& Ross, 2006), it makes sense that mothers, who feel less support from their own parents may also experience more PPDS, as was the case for the Czech mothers. Social support is not a unidimensional construct, and the type of support (emotional, instrumental, informational, appraisal) varies by source. It is possible that the type of support that the mothers received from their own parents differed between the two cultures. It is also possible that receiving more parental support, which may be more of a cultural expectation in Asian cultures where mothers and female family relatives have traditionally been involved postpartum care (Goyal, Park, \& McNiesh, 2015; Kaewsarn et al., 2003), buffered against depressive symptoms for the Thai mothers. Such expectations of familial support are not often as prevalent in westernized nations so perhaps the Czech women's parents' involvement and the type of support provided differed in the postpartum period. Future research can continue to explore the type of postpartum support received from family, friends,

Regardless of the country in which a new mother lives, the identification of risk factors may assist in earlier detection and intervention to reduce PPDS severity. and the community among women living in different countries.

We believe that the current study provides useful information about the postpartum well-being of Czech and Thai mothers (see Table 3); however, several limitations should be noted. First, the measures were translated and back-translated from English to Czech and Thai; however, there has been limited PPDS research in these countries and the measures have not been validated in these languages, except for (Pitanupong et al., 2007) validation of the EDPS with Thai mothers. Also, cultural practices differ widely between the Czech Republic and Thailand and the women who live in these countries give birth in different settings with disparate health care available to them. Moreover, the women in both countries were self-selected, and nearly all were married or in a committed relationship. Many had additional children (multiparous) and had a college level education or higher. The results, therefore, may not be generalizable to women who have different demographic characteristics. Additionally, although previous researchers have found psychiatric history, particularly prenatal depressive symptoms, to be an important risk factor, depression history was not considered in either regression model as too few women in either sample reported prior depressive symptoms. These variables should be considered with larger sample sizes.

Despite the limitations, this study has important implications for childbirth educators working with mothers and their families. As the current findings suggest overlap in some risk factors for women across the globe, prevention programs that have been successful in reducing PPDS in one country may be both relevant and helpful to mothers living elsewhere in the world. Both researchers and clinicians, of course, must take cultural context into consideration when 
working with mothers to implement such programs (Gulamani, Premji, Kanji, \& Azam, 2013). Culturally targeted interdisciplinary interventions should be used to help mothers and their families become more aware of common risks for PPDS and teach them how to modify, or reduce, these risk factors whenever possible. Certain interventions could include organizing community support groups aimed at bolstering maternal efficacy, normalizing making mistakes (targeting perfectionism), minimizing partner conflict, and identifying ways to cope with stressors such as financial burden. Regardless of the country in which a new mother lives, the identification of risk factors may assist in earlier detection and intervention to reduce PPDS severity. Such preventive interventions can continue to have a positive impact on women's lives and emotional well-being while reducing the cost of health care.

\section{REFERENCES}

Al Dallal, F. H., \& Grant, I. N. (2012). Postnatal depression among Bahraini women: Prevalence of symptoms and psychosocial risk factors. Eastern Mediterranean Health Journal, 18(5), 432-438. http://dx.doi.org/10.26719/ 2012.18.5.432

Bartonova, D. (2005). Trends in census households in the Czech Republic in the last third of the 20th century. Demografie, 47, 1-12.

Callister, L. C., Beckstrand, R. L., \& Corbett, C. (2010). Postpartum depression and culture: Pesado Corazon. MCN. The American Journal of Maternal Child Nursing, 35(5), 254-261. http://dx.doi.org/10.1097/ NMC.0b013e3181e597bf

Caramlau, I., Barlow, J., Sembi, S., McKenzie-McHarg, K., \& McCabe, C. (2011). Mums 4 Mums: Structured telephone peer-support for women experiencing postnatal depression. Pilot and exploratory RCT of its clinical and cost effectiveness. Trials, 12, 88-93. http://dx.doi. org/10.1186/1745-6215-12-88

Chaaya, M., Campbell, O. M., El Kak, F., Shaar, D., Harb, H., \& Kaddour, A. (2002). Postpartum depression: Prevalence and determinants in Lebanon. Archives of Women's Mental Health, 5(2), 65-72. http://dx.doi.org/ 10.1007/s00737-002-0140-8

Choi, S. Y., Kim, E. J., Ryu, E., Chang, K. O., \& Park, M. N. (2012). Postpartum depression and parental self-efficacy: A comparison of native Korean and Vietnamese immigrant mothers in Korea. Journal of Transcultural Nursing : Official Journal of the Transcultural Nursing Society, 23(2), 181-187. http://dx.doi.org/10.1177/ 1043659611434057

Cox, J. L., Holden, J. M., \& Sagovsky, R. (1987). Detection of postnatal depression. Development of the 10-item Edinburgh postnatal depression scale. The British Journal of Psychiatry, 150, 782-786. http://dx.doi.org/ 10.1192/bjp.150.6.782 de Graaf, L. E., Roelofs, J., \& Huibers, M. J. (2009). Measuring dysfunctional attitudes in the general population: The dysfunctional attitude scale (form A) revised. Cognitive Therapy and Research, 33(4), 345355. http://dx.doi.org/10.1007/s10608-009-9229-y

Dennis, C. L., \& Ross, L. (2006). Women's perceptions of partner support and conflict in the development of postpartum depressive symptoms. Journal of Advanced Nursing, 56(6), 588-599. http://dx.doi.org/10.1111/j. 1365-2648.2006.04059.x

Dindar, I., \& Erdogan, S. (2007). Screening of Turkish women for postpartum depression within the first postpartum year: The risk profile of a community sample. Public Health Nursing, 24(2), 176-183. http:// dx.doi.org/10.1111/j.1525-1446.2007.00622.x

Dudas, R. B., Csatordai, S., Devosa, I., Töreki, A., Andó, B., Barabás, K., ... Kozinszky, Z. (2012). Obstetric and psychosocial risk factors for depressive symptoms during pregnancy. Psychiatry Research, 200(2-3), 323-328. http://dx.doi.org/10.1016/j.psychres.2012.04.017

Erdfelder, E., Faul, F., \& Buchner, A. (1996). GPOWER: A general power analysis program. Behavior Research Methods, Instruments, \& Computers, 28(1), 1-11. http://dx.doi.org/10.3758/BF03203630

Gaillard, A., Le Strat, Y., Mandelbrot, L., Keïta, H., \& Dubertret, C. (2014). Predictors of postpartum depression: Prospective study of 264 women followed during pregnancy and postpartum. Psychiatry Research, 215(2), 341-346. http://dx.doi.org/10.1016/j.psychres. 2013.10.003

Gao, L. L., Chan, S. W., \& Sun, K. (2012). Effects of an interpersonal-psychotherapy-oriented childbirth education programme for Chinese first-time childbearing women at 3-month follow up: Randomised controlled trial. International Journal of Nursing Studies, 49(3), 274-281. http://dx.doi.org/10.1016/j.ijnurstu.2011.09.010

Gibaud-Wallston, J., \& Wandersman, LP. (1978). "Development and utility of the Parenting Sense of Competence Scale." Paper presented at the annual meeting of the American Psychological Association, Toronto.

Goyal, D., Park, V. T., \& McNiesh, S. (2015). Postpartum depression among Asian Indian mothers. MCN, The American Journal of Maternal/Child Nursing, 40(4), 256-261. http://dx.doi.org/10.1097/NMC.0000000000 000146

Grote, N. K., \& Bledsoe, S. E. (2007). Predicting postpartum depressive symptoms in new mothers: The role of optimism and stress frequency during pregnancy. Health \& Social Work, 32(2), 107-118. http://dx.doi. org/10.1093/hsw/32.2.107

Gulamani, S. S., Premji, S. S., Kanji, Z., \& Azam, S. I. (2013). A review of postpartum depression, preterm birth, and culture. The Journal of Perinatal \& Neonatal Nursing, 27(1), 52-59.

Halbreich, U., \& Karkun, S. (2006). Cross-cultural and social diversity of prevalence of postpartum depression and depressive symptoms. Journal of Affective Disorders, 91(2-3), 97-111. http://dx.doi.org/10.1016/ j.jad.2005.12.051 
Hammen, C. (2003). Interpersonal stress and depression in women. Journal of Affective Disorders, 74(1), 49-57. http://dx.doi.org/10.1016/S0165-0327(02)00430-5

Haslam, D. M., Pakenham, K. I., \& Smith, A. (2006). Social support and postpartum depressive symptomatology: The mediating role of maternal self-efficacy. Infant Mental Health Journal, 27(3), 276-291. http://dx.doi. org/10.1002/imhj.20092

Hassert, S., Kurpius, S. E., \& Tracey, T. J. (2015). Testing a conceptual model of postpartum depressive symptoms in the first year. Women \& Health, 55(6), 700-716. http://dx.doi.org/10.1080/03630242.2015. 1039181

Hassert, S., \& Robinson Kurpius, S. E. (2011). Postpartum depression among Latinas: Do partners, other children, and breastfeeding make a difference? Journal of Multicultural Counseling \& Development, 39, 90 100.

Hopkins, J., \& Campbell, S. B. (2008). Development and validation of a scale to assess social support in the postpartum period. Archives of Women's Mental Health, 11(1), 57-65. http://dx.doi.org/10.1007/s00737 $-008-0212-5$

Hraba, J., Lorenz, F. O., \& Pechačová, Z. (2000). Family stress during the Czech transformation. Journal of Marriage and Family, 62(2), 520-531. http://dx.doi. org/10.1111/j.1741-3737.2000.00520.x

Kaewsarn, P., Moyle, W., \& Creedy, D. (2003). Traditional postpartum practices among Thai women. Journal of Advanced Nursing, 41(4), 358-366. http://dx.doi.org/ 10.1046/j.1365-2648.2003.02534.x

Kim, Y. K., Hur, J. W., Kim, K. H., Oh, K. S., \& Shin, Y. C. (2008). Prediction of postpartum depression by sociodemographic, obstetric and psychological factors: A prospective study. Psychiatry and Clinical Neurosciences, 62(3), 331-340. http://dx.doi.org/10. 1111/j.1440-1819.2008.01801.x

Klainin, P., \& Arthur, D. G. (2009). Postpartum depression in Asian cultures: A literature review. International Journal of Nursing Studies, 46(10), 1355-1373. http:// dx.doi.org/10.1016/j.ijnurstu.2009.02.012

Lanes, A., Kuk, J. L., \& Tamim, H. (2011). Prevalence and characteristics of postpartum depression symptomatology among Canadian women: A cross-sectional study. BMC Public Health, 11, 302. http://dx.doi.org/ 10.1186/1471-2458-11-302

Liabsuetrakul, T., Vittayanont, A., \& Pitanupong, J. (2007). Clinical applications of anxiety, social support, stressors, and self-esteem measured during pregnancy and postpartum for screening postpartum depression in Thai women. Journal of Obstetrics and Gynaecology Research, 33(3), 333-340. http://dx.doi.org/10.1111/j. 1447-0756.2007.00532.x

Limlomwongse, N., \& Liabsuetrakul, T. (2006). Cohort study of depressive moods in Thai women during late pregnancy and 6-8 weeks of postpartum using the Edinburgh Postnatal Depression Scale (EPDS). Archives of Women's Mental Health, 9(3), 131-138. http://dx.doi.org/10.1007/s00737-005-0115-7
Marcus, S. M. (2009). Depression during pregnancy: rates, risks and consequences-Motherisk Update 2008. The Canadian Journal of Clinical Pharmacology = Journal Canadien de Pharmacologie Clinique, 16(1), 15-22.

Milgrom, J., Ericksen, J., \& Sved-Williams, A. (2016). Impact of parental psychiatric illness on infant development. In A. -L. Sutter-Dallay, N. M. -C. GlangeaudFreudenthal, A, A. Guedeney, \& A. Riecher-Rossler (Eds.), Joint care of parents and infants in perinatal psychiatry (pp. 47-78). Switzerland: Springer International.

Milgrom, J., Gemmill, A. W., Bilszta, J. L., Hayes, B., Barnett, B., Brooks, J., . . . Buist, A. (2008). Antenatal risk factors for postnatal depression: A large prospective study. Journal of Affective Disorders, 108(1-2), 147157. http://dx.doi.org/10.1016/j.jad.2007.10.014

Nishioka, E., Haruna, M., Ota, E., Matsuzaki, M., Murayama, R., Yoshimura, K., \& Murashima, S. (2011). A prospective study of the relationship between breastfeeding and postpartum depressive symptoms appearing at 1-5 months after delivery. Journal of Affective Disorders, 133(3), 553-559. http://dx.doi.org/10.1016/ j.jad.2011.04.027

Oates, M. R., Cox, J. L., Neema, S., Asten, P., GlangeaudFreudenthal, N., Figueiredo, B., . . . Yoshida, K.., \& TCS-PND Group. (2004). Postnatal depression across countries and cultures: A qualitative study. The British Journal of Psychiatry, 46, s10-s16. http://dx.doi.org/10. 1192/bjp.184.46.s10

Pierce, G. R., Sarason, I. G., \& Sarason, B. R. (1991). General and relationship-based perceptions of social support: Are two constructs better than one? Journal of Personality and Social Psychology, 61(6), 1028-1039. http://dx.doi.org/10.1037/0022-3514.61 .6 .1028

Pitanupong, J., Liabsuetrakul, T., \& Vittayanont, A. (2007). Validation of the Thai Edinburgh postnatal depression scale for screening postpartum depression. Psychiatry Research, 149(1-3), 253-259. http://dx.doi.org/10. 1016/j.psychres.2005.12.011

Segre, L. S., O'Hara, M. W., Arndt, S., \& Stuart, S. (2007). The prevalence of postpartum depression: The relative significance of three social status indices. Social Psychiatry and Psychiatric Epidemiology, 42(4), 316321. http://dx.doi.org/10.1007/s00127-007-0168-1

Takács, L., Smolík, F., Mlíková Seidlerová, J., Čepický, P., \& Hoskovcová, S. (2016). [Postpartum blues - a Czech adaptation of the Maternity Blues Questionnaire]. Ceska Gynekologie, 81(5), 355-368.

Vliegen, N., Casalin, S., \& Luyten, P. (2013). The course of postpartum depression: A review of longitudinal studies. Harvard Review of Psychiatry, 22, 1-22.

Vliegen, N., Luyten, P., Besser, A., Casalin, S., Kempke, S., \& Tang, E. (2010). Stability and change in levels of depression and personality: A follow-up study of postpartum depressed mothers that were hospitalized in a mother-infant unit. The Journal of Nervous and Mental Disease, 198(1), 45-51. http://dx.doi.org/10.1097/ NMD.0b013e3181c8aa57 
Whittaker, A. (2002). Water serpents and staying by the fire: Markers of maturity in a northeast Thai village. In L. Manderson \& P. Liamputtong (Eds.), Coming of age in South and Southeast Asia: Youth, courtship, and sexuality (pp. 17-41). Richmond, Australia: Curzon Press.

Xie, R. H., He, G., Koszycki, D., Walker, M., \& Wen, S. W. (2009). Prenatal social support, postnatal social support, and postpartum depression. Annals of Epidemiology, 19(9), 637-643. http://dx.doi.org/10. 1016/j.annepidem.2009.03.008
SILVA HASSERT is a licensed psychologist at Kaiser Permanente in Northern California. SHARON ROBINSON KURPIUS is a licensed psychologist and tenure-track faculty member at Arizona State University in the Counseling Psychology Department. ANUSORN PAYAKKAKOM is a faculty member in the Department of Social Science at Mahidol University in Thailand. ELIŠKA KODYŠOVÁ is a research specialist for the Healthy Parenting Association in the Czech Republic. 